Marko Švaco

Nikola Vitez

Bojan Šekoranja $\bowtie$

Filip Šuligoj

https://doi.org/10.21278/TOF.42302

ISSN 1333-1124

eISSN 1849-1391

\title{
TUNING OF PARAMETERS FOR ROBOTIC CONTOURING BASED ON THE EVALUATION OF FORCE DEVIATION
}

\begin{abstract}
Summary
The application of industrial robots with advanced sensor systems in unstructured environments is continuously becoming wider. A widely used type of advanced sensor systems is the force-torque sensor. Force-torque sensors are typically used for applications such as robot grinding, sanding, polishing, and deburring, where a constant force is exerted upon a workpiece. In this research, control parameters for exerting a constant force along a predefined path are evaluated in laboratory conditions. The experimental setup with the contouring force feedback is composed of a Fanuc LRMate six-degree-of-freedom industrial robot with an integrated force-torque sensor. Control parameters of the Contouring function within the Fanuc robot controller are tuned in four contouring experiments. The experiments conducted in this research are: i) flat beam, ii) flat beam with a rigid support, iii) wave shaped compliant plate, and iv) compliant flat plate. During the experiments, contouring parameters were altered in order to collect the feedback on the values of the force to be used for the evaluation of the force deviation. A fitness function for the evaluation of the force deviation and the tuning of the control parameters is presented. The fitness function enables a selection of initial control parameters which minimize the force deviation during the robot contouring process.
\end{abstract}

Key words: $\quad$ robotics, force-torque sensor, force sensing, contouring

\section{Introduction}

To reduce the percentage of manual labor in industrial processes, and, consequently, to increase productivity and product quality, these processes should be fully automated. For efficient automation, it is necessary to provide a highly organized working environment or to integrate robots with advanced sensor systems that allow operation in unstructured environments [1]. Furthermore, in applications such as grinding or polishing people work under harsh working conditions; this certainly justifies automation. In modern production systems, the integration between advanced sensor systems and robotic technology is preferred because it allows easy adaptation to different tasks when there is a high probability of product changes, and because of the introduction of the mass customization instead of the mass production principle. Some of the advanced sensor systems that can be integrated with robotic systems include 2D and 3D vision systems [2], force and torque sensors [3] or special 
capacitive sensors that cover the whole robot body (having a function of detecting the presence of human workers) [4]. Today, industrial robotics is facing the challenge of high accuracy demands. Responding to that challenge, the robotics community is continuously developing versatile methods for robot calibration or correction [5], [6]. In this paper, we develop and validate an application with a force-torque sensor for a contouring task. One of the main benefits of using force sensors in robot applications is the ability to adapt to changing conditions, such as part position and orientation and also part geometry. Adaptivity of robotic systems with force feedback prevents excessive contact forces and enables a consistent contact force to be obtained through force regulation [7].

A constant normal force applied to the surface is a basic requirement for machining tasks such as polishing and grinding. There are two basic approaches to maintaining a constant force on a surface. The first one implies applying a force by changing the position of the robot axes. The second one uses the robot for coarse positioning while an auxiliary end effector is used for maintaining a constant force [8]. Machining tasks are highly demanding for standard industrial robots because of their non-rigid kinematic structure. There are three basic parameters that affect the stiffness of the robot structure: joint stiffness, robot configuration, and the exerted force. One way to maximize the stiffness of the robot structure is to optimize the robot configuration because it is the only parameter which can be set arbitrarily in contouring applications [9]. Tian et al. optimized the layout and the operational configuration of the robot for the grinding application based on the optimal stiffness obtained by means of a genetic algorithm. The dynamic behaviour of a robot can be affected by active stiffness imposed by the controller as discussed in [10]. Bonilla et al. developed a control strategy for contouring carried out by industrial robots based on an uncalibrated vision sensor for path planning and a force/torque sensor for impedance control. The developed control strategy allows the contouring of workpieces of unknown location and geometry. In practice, a complete dynamic model of a robot cannot be established and the environment stiffness cannot be estimated accurately, which causes the system performance degradation [11]. Part tolerances and errors that occur in positioning influence the robot accuracy, which is further affected by the part and the tool wear. Robot applications utilizing force control reduce the previously mentioned errors. The ability to adapt to undefined geometry reduces the time needed for programming due to a lower degree of precision required in the teaching of the trajectory points [12]. Sudden changes in the part geometry usually cause high force oscillations and even the separation between the tool and the workpiece during contouring. This problem is successfully addressed in [13] where the feed rate is controlled as a function of force control error. In a study conducted by Tahvilian et al. [14], a robotic grinding force model based on an idealized uncut chip for the on-site maintenance of hydropower equipment is developed. In [15], a novel methodology for robotic belt grinding is proposed. The primary focus is on the system calibration and force control to improve the performance in belt grinding procedures. A fuzzybased control system is proposed in [16] for robotic grinding and polishing. Zhang et al. [17] developed a hybrid passive and active force control system for grinding with an industrial robot. An abrasive belt grinder with dynamic position adjustment was developed. The grinding process is planned and simulated in V-REP and afterward executed on the actual robot. Regarding more complicated tasks such as robotic polishing, a set of compliant tools were developed and used for the polishing of aspheric surfaces [18]. Zamri et al. conducted a polishing experiment on a Comau robotic cell with input parameters that included grit size, polishing time, and polishing force. Their experimental results reveal that polishing time and grit size have the highest impact on surface roughness [19]. Optimization of robotic polishing parameters (force, feed rate, and rotational speed of the tool) by using a neural network and a genetic algorithm proved to be very effective in achieving the desired material removal and surface roughness improvement on uneven surfaces [20]. Du et al. developed an automatic 
robotic polishing technique implemented in the system for polishing curved parts made of a titanium alloy. The system is composed of a specially designed end effector, a fuzzy force controller, and a software solution for the correction of polishing trajectory. It uses the force sensor data acquired after every cycle and gives a satisfactory surface quality [21]. In the absence of force and torque sensors, either compliant robot tools [22] or active robot flanges [23] can be used for the compensation of small part irregularities and geometry differences. A novel design of a robot end effector is proposed in [24] to provide the ability of dampening the forces exerted on the robot arm. Another end effector based on the fusion of a force and a vision sensor is developed in [25]; it provides high-speed robotic contour tracing and enables the teaching of trajectory points.

The main objective of this paper is to validate the possibilities of robotic contouring with force feedback in applications where a constant normal force along a spatial trajectory needs to be exerted on the part surface by using an analytical evaluation of the force contouring parameters based on force deviation. In this paper, we provide extensive experimental results and propose force contouring parameters for different part geometries in four contouring experiments with: i) flat beam, ii) flat beam with a rigid support, iii) wave shaped compliant plate, and iv) compliant flat plate. For each test, the paper provides control parameters determined on the basis of fitness function which minimizes errors in the force exerted by the robot. The paper is an extension of our previous study, [26], in which we evaluated the contouring parameters qualitatively, without an objective function. In this paper, we propose a novel objective fitness function for the calculation and validation of the best contouring parameters for a given case. We have also introduced an additional test (horizontal beam without additional support) to evaluate the environment stiffness effect on the force deviation during contouring.

\section{Materials and methods}

\subsection{System setup}

The experimental setup consists of a FANUC LRMate $200 \mathrm{iC} / 5 \mathrm{~L}$, the 6 degree-offreedom industrial robot with an integrated Fanuc FS-10iA force-torque sensor. The force sensor allows the measurement of forces and moments with respect to the three coordinate axes (Fx, Fy, Fz, Mx, My, Mz). The rated load of the sensor is $98 \mathrm{~N}$, with a resolution of 0.39 $\mathrm{N}$ (the reading error is below $2 \%$ ). We have designed and manufactured a robot tool shown in Figure 1 to be used for exerting a constant normal force on the workpiece surface. The tool is composed of two ball bearings on a shaft attached to an aluminum plate. The outer rings of the ball bearings are in contact with the workpiece surface during contouring. The overall weight of the tool and the force sensor assembly connected to the robot flange is $2.16 \mathrm{~kg}$.
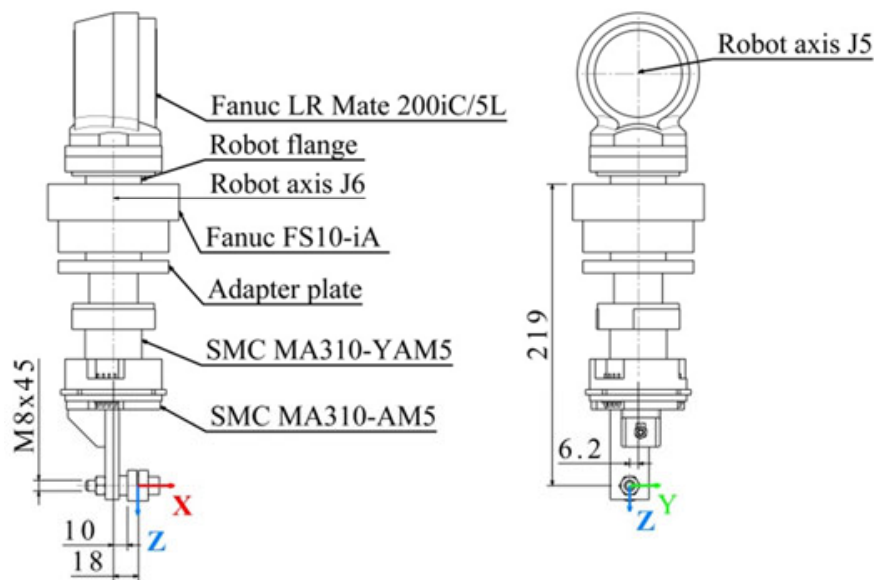

Fig. 1 Tool centre point (TCP) and tool coordinate system shown on the robot contouring tool assembly 
The contouring tests include a flat beam (aluminium rail) used with a rigid support (end point 2) and without a rigid support (end point 1), shown in Figure 2, a wave-shaped compliant plate, shown in Figure 3, and a compliant flat plate, shown in Figure 4. The compliant plates have a soft insert between the rigid plate and the work table to reduce vibrations of the robotic system.

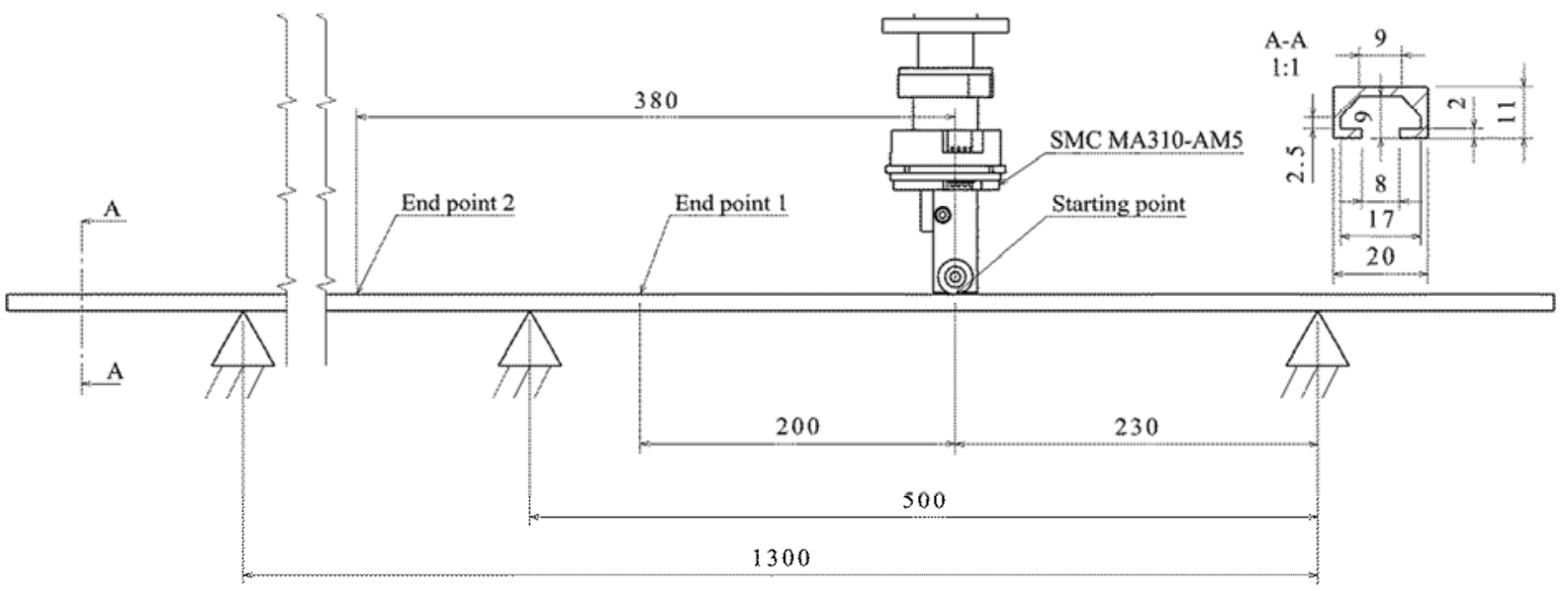

Fig. 2 Flat beam experiment

Figure 3 shows a detailed representation of the starting and end points used for contouring on the wave shaped plate.

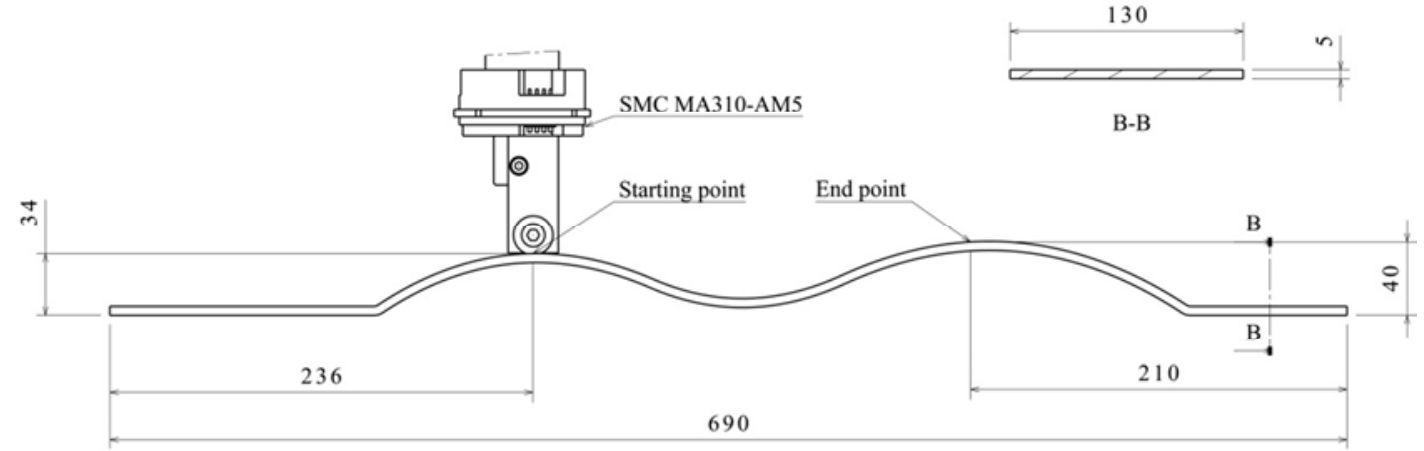

Fig. 3 Wave shaped plate experiment
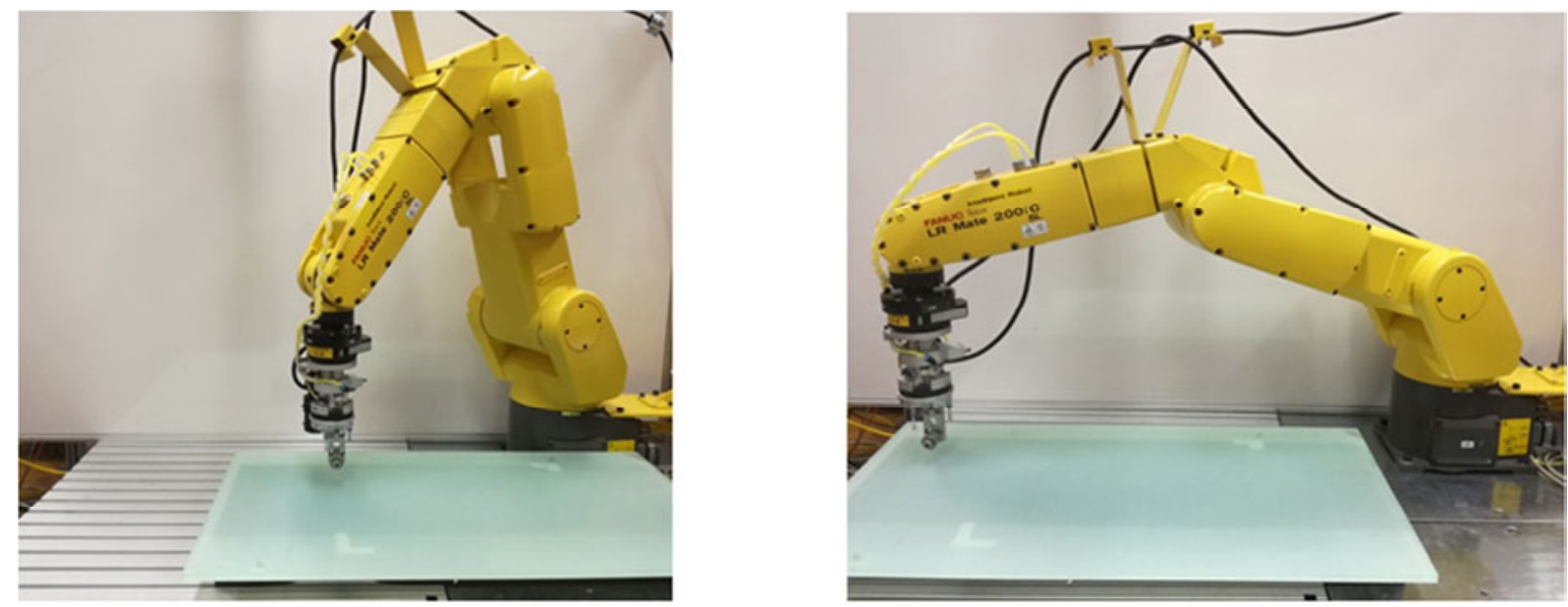

Fig. 4 Test iv) -a compliant flat plate in different parts of the robot workspace; the rigid plexiglass plate is placed on a deformable rubber plate 


\subsection{Robot contouring}

The robot contouring application traces the surface of a workpiece while maintaining a constant force in a predefined direction. A combination of robot contouring application and an adequate robot tool enables polishing or grinding. During the execution of robot contouring operation, a constant force is applied along an approximate path determined from the points stored as ordinary motion statements. The points required for a normal execution of the robot contouring are: a starting point, an end point, and intermediate points where the robot posture changes radically due to the curvature of the traced surface. The taught points should be set so that the tool centre point (TCP) is about $1 \mathrm{~mm}$ away from the surface of the workpiece because if it were not the case, an excessive force could be applied. The taught points are also set so that the pushing direction (Z-axis of the robot TCP coordinate system) is always collinear with the surface normal. It is important to perform gravity compensation as part of robot contouring since the gravity acting on the force sensor differs with changes in robot posture [26]. The main parameters which were used and tuned in our experiments for robot contouring are:

- Contact F Threshold - the threshold for determining contact made with the workpiece. The actual contouring operation starts after this contact is made,

- Approach Velocity - speed used in the pushing direction until a contact with the workpiece is made

- Control Frame - determines whether to perform pushing in the tool frame or the user frame. The user frame is used for pushing a workpiece in a constant direction (the workpiece is held by a robot) and the tool frame is used in applications where pushing direction varies (tool on the robot flange),

- Contouring Force - specifies a target pushing force to be used

- Force Control Gain - determines a response during the force control. The settings of this parameter are critical for successful force control without vibrations of the robotic system. The force control gain setting is not the same in the phase while approaching the workpiece (Approach Impedance parameter) as in the phase of contouring execution (Contouring Impedance parameter). There are two options for the tuning of impedance parameters:

- Master frequency - determines the response in the direction of pushing;

- Individual frequency - determines the response on the direction-by-direction basis;

- Pushing Direction Velocity - specifies the speed in the direction of pushing during contouring. This speed needs to be specified to prevent the tool moving away from the workpiece due to the large curvature of the workpiece.

Contact F Threshold, Approach Velocity, and Approach Impedance determine the initial transient response. These parameters were tuned empirically and kept constant during experiments. The Contouring Impedance parameter determines, through the tuning of the Individual frequency, the response oscillations and the robot compliance after the initial contact is made; therefore, it is the key to good contouring performance.

The process of the contouring experiment conducted for the flat beam is shown in Figure 5. Measurement results used for the calculation and validation of parameters for the tests i) and ii) are those collected between points shown in Figures $5 \mathrm{~b}$ and $5 \mathrm{~d} / \mathrm{e}$. 
M. Švaco, N. Vitez,

B. Šekoranja, F. Šuligoj

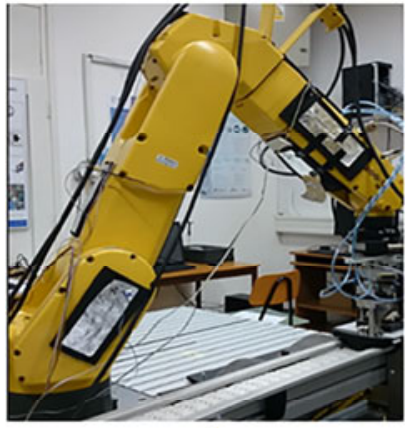

a)

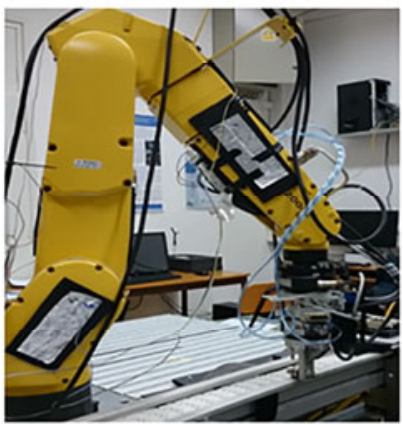

d)

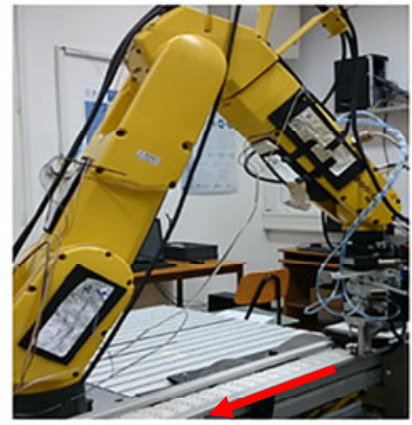

b)

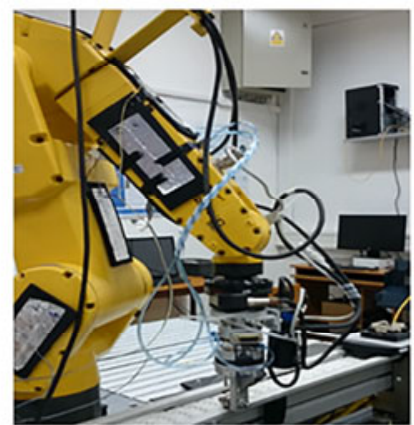

e)

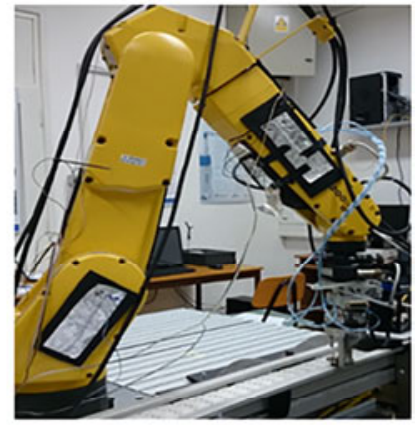

c)

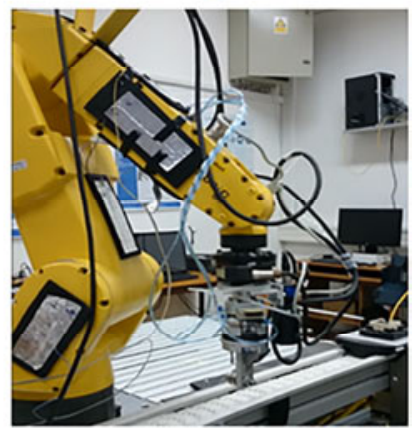

f)

Fig. 5 Contouring experiment for the flat beam, cases i) and ii): a) approaching point, b) start of contouring with the defined force; the red arrow shows the direction of the contouring path, c) contouring along the path approximated by taught points, d) contouring end point for case i), e) contouring end point for case ii), f) moving away from the beam surface

\section{Results}

In order to collect data on robotic contouring with a constant normal force applied, a series of experiments were conducted with different forces and feed rates applied along predefined paths. The Force Control Gain parameters were fine-tuned in order to obtain experiment-based solutions and to examine their impact on robot contouring.

The robot payload $(5 \mathrm{~kg})$ has been taken into account during the selection of the force exerted on the workpiece by the tool. Based on the robot payload and the mass of the tool assembly $(2.16 \mathrm{~kg})$ we have concluded that the maximum permissible magnitude of the exerted force is $28 \mathrm{~N}$. Considering the maximum permitted magnitude of force and the initial experiments, four values of forces were selected for obtaining experimental data, $F_{1}=10 \mathrm{~N}$, $F_{2}=15 \mathrm{~N}, F_{3}=20 \mathrm{~N}$, and $F_{4}=25 \mathrm{~N}$. Feed rates for grinding and polishing range from a few millimetres per minute up to several metres per minute. Considering the wide range of possible feed rates $\left(\mathrm{v}_{\mathrm{s}}\right)$, their selection along predefined paths was based on the experimental tests. During the selection of feed rates the working frequency of the robot controller $(125 \mathrm{~Hz})$ was also taken into account to ensure that the robot can do at least one correction of the force applied per millimetre of the path. Considering the previously mentioned criteria, feed rates of $40,50,60,70,75,80,90,100$, and $110 \mathrm{~mm} / \mathrm{s}$ were selected for measurements on the flat beam. Feed rates of $5,10,20,25,30,35,40,50$, and $60 \mathrm{~mm} / \mathrm{s}$ were selected for measurements in the more demanding case of the wave-shaped plate.

\subsection{Tuning of the robot contouring parameters}

Since the Contouring Impedance parameter is the key to a good performance of robot contouring, we have decided to adjust the individual frequency for each axis of the tool coordinate system. This way, only the response in the direction normal to the surface (in this case the Z-axis of the tool coordinate system) is affected. Initial frequencies for translations 
and rotations around the robot tool axes, both for the flat beam and the wave formed plate, are given in Table 1. Frequencies for the Z-axis direction were altered while the values of frequencies for the remaining translations and rotations were kept constant. The rest of the tuned robot contouring parameters are shown in Table 2. All the other robot contouring parameters were left at their default settings.

Table 1 Robot contouring individual frequencies

\begin{tabular}{|c|c|c|c|}
\hline & Flat beam & Wave-formed plate & Compliant flat plate \\
\hline$f_{\mathrm{z}}[\mathrm{Hz}]$ & $1,3,5,7,10$ & $5,10,15,20,25$ & 5 \\
\hline$f_{\mathrm{x}}=f_{\mathrm{y}}[\mathrm{Hz}]$ & 0.5 & 0.007 & 0.5 \\
\hline$f_{\mathrm{w}}=f_{\mathrm{p}}=f_{\mathrm{r}}[\mathrm{Hz}]$ & 0.349 & 0.0005 & 0.349 \\
\hline
\end{tabular}

Table 2 Constant contouring parameters [26]

\begin{tabular}{|c|c|c|}
\hline Case & $i, i i, i v$ & $i i i$ \\
\hline Pushing Direction & $+\mathrm{Z}$ & $+\mathrm{Z}$ \\
\hline Contact $F$ Threshold [N] & $\begin{array}{l}1\left(F_{1}=10 ; F_{2}=15\right), \\
2\left(F_{3}=20 ; F_{4}=25\right)\end{array}$ & $\begin{array}{l}1\left(F_{1}=10 ; F_{2}=15\right), \\
2\left(F_{3}=20 ; F_{4}=25\right)\end{array}$ \\
\hline Approach Velocity [mm/s] & 10 & 1 \\
\hline Control Frame & Tool Frame & Tool Frame \\
\hline Contouring Force $[\mathrm{N}]$ & $\begin{array}{c}F_{1}=10 ; F_{2}=15 ; F_{3}=20 ; \\
F_{4}=25\end{array}$ & $\begin{array}{c}F_{1}=10 ; F_{2}=15 ; F_{3}=20 ; \\
F_{4}=25\end{array}$ \\
\hline Approach Impedance [Hz] & $\begin{array}{c}f_{\mathrm{x}}=f_{\mathrm{y}}=0.05, f_{\mathrm{z}}=1, \\
f_{\mathrm{w}}=f_{\mathrm{p}}=f_{\mathrm{r}}=0.349\end{array}$ & $\begin{array}{c}f_{\mathrm{x}}=f_{\mathrm{y}}=0.007, f_{\mathrm{z}}=1, \\
f_{\mathrm{w}}=f_{\mathrm{p}}=f_{\mathrm{r}}=0.349\end{array}$ \\
\hline $\begin{array}{l}\text { Pushing Direction Velocity } \\
{[\mathrm{mm} / \mathrm{s}]}\end{array}$ & 15 & 1 \\
\hline
\end{tabular}

\subsection{Selection of experimental frequency}

The experimental frequency for a given feed rate with a defined exerted force is determined by eliminating all frequencies with the values of $\Delta F_{\text {error }}$ higher than $25 \mathrm{~N}$, where $\Delta F_{\text {error }}=F_{\max }-F_{\min }$. A high value of $\Delta F_{\text {error }}$ always causes significant and undesirable vibrations of the robotic system. Another criterion which includes frequencies with minimum variations around the nominal value is determined by the equation (1), where $F_{\text {std }}$ is the standard deviation of the mean value of the exerted force $F_{\text {mean }}$ :

$$
F_{\text {std }}=\sqrt{\frac{1}{N} \sum_{i=1}^{N}\left|F_{i}-F_{\text {mean }}\right|^{2}} .
$$

The next criterion is the minimum difference between the nominal value of force $F_{0}$ and the mean value of the exerted force $F_{\text {mean }}$ given by $\Delta F=\left|F_{0}-F_{\text {mean }}\right|$. The fitness of each frequency for a given feed rate and exerted force is calculated by normalizing each criterion and adding up normalized values. The first minimum value for each criterion $\left(\min \left(\Delta F_{\text {error }}\right)\right.$, $\min \left(F_{\text {std }}\right)$, and $\left.\min (\Delta F)\right)$ is determined for all the frequencies applied for a given feed rate and force. After that, the criteria for each frequency are normalized by the following equations:

$$
\begin{aligned}
& f_{\text {std }}(n)=\min \left(F_{\text {std }}\right) / F_{\text {std }}(n), \\
& \Delta f_{\text {error }}(n)=\min \left(\Delta F_{\text {error }}\right) / \Delta F_{\text {error }}(n), \\
& \Delta f(n)=\min (\Delta F) / \Delta F(n),
\end{aligned}
$$


where $n$ is the applied frequency. The fitness of each frequency is determined by the following equation:

$$
f_{n}=f_{\text {std }}(n)+\Delta f_{\text {error }}(n)+\Delta f(n) .
$$

The frequency with a higher value of fitness is better for a given feed rate and force. The criteria (steps) for the determination of frequency fitness are shown in Figure 6. The selection of optimal frequencies is evaluated based on the graphs showing the fitness for each frequency for all the feed rates applied and forces exerted; they are shown in Figure 7 and Figure 8 .

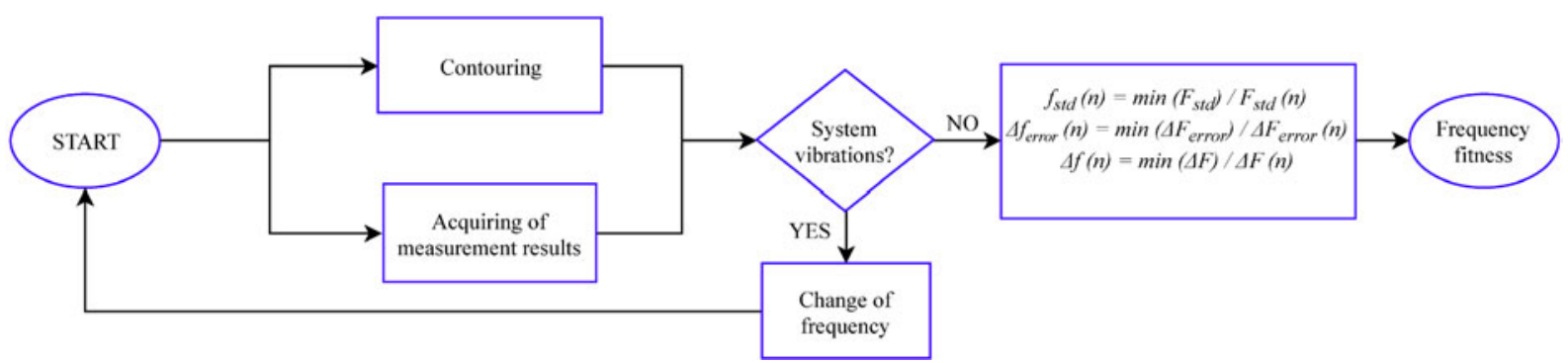

Fig. 6 Flowchart for calculating the fitness of a frequency

\subsection{Flat beam test, cases i) and ii)}

Possibilities of the application of a constant normal force, exerted by a robot, along flat surfaces and a force sensor were investigated for force reference values of $10 \mathrm{~N}, 15 \mathrm{~N}, 20 \mathrm{~N}$, and $25 \mathrm{~N}$. The deviation of the applied force was observed for the feed rates of 40, 50, 60, 70, $75,80,90,100$, and $110 \mathrm{~mm} / \mathrm{s}$. Tests on the flat beam were conducted for two cases: i) contouring end point 1 before the rigid support and ii) contouring end point 2 after the support, both shown in Figure 2.

After the experimental tests for the case i) had been conducted, with all the frequency changes along the Z-axis of the tool coordinate system for each feed rate and force, the fitness of the frequencies for all the cases was calculated. Graphs in Figure 7 show the fitness for frequencies of 3,5, and $7 \mathrm{~Hz}$ for all the feed rates applied and forces exerted. Mean fitness values for frequencies of 3,5 , and $7 \mathrm{~Hz}$ are 2.15, 2.52, and 2.61, respectively. Regarding the mean fitness values, the frequency of $7 \mathrm{~Hz}$ in the Z-axis direction is a good choice for the tuning of the Contouring impedance parameter in cases similar to the case i). This frequency gives the best response with minimum vibrations of the robotic system for almost all the values of the force and feed rates used in the experiments.
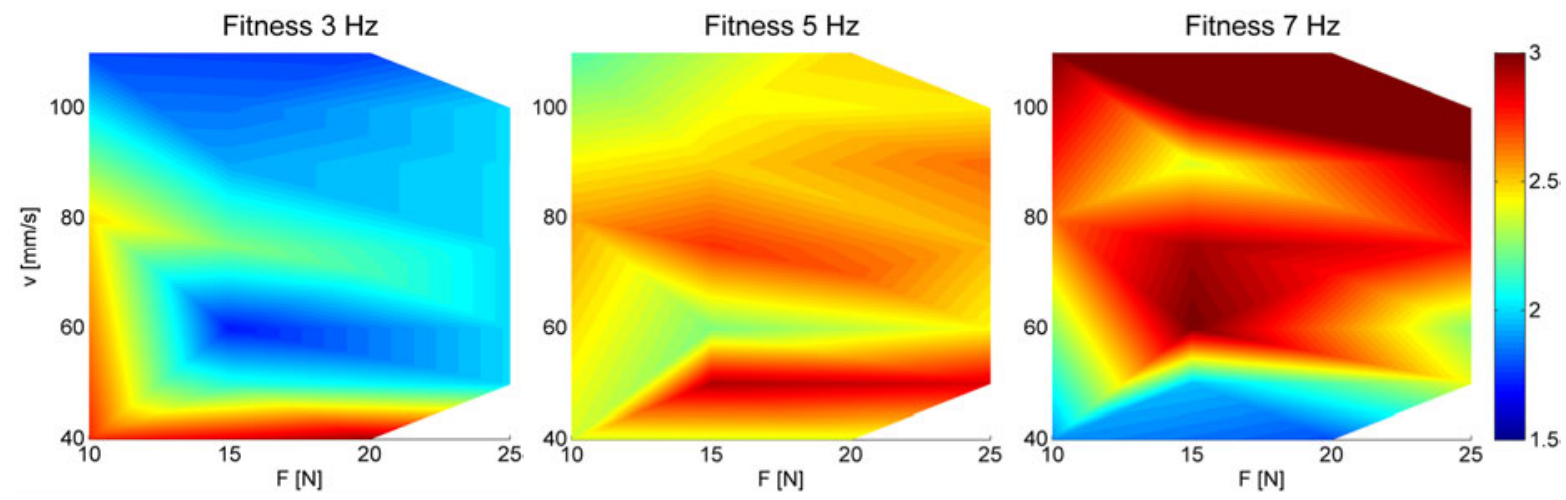

Fig. 7 Fitness of frequencies for the flat beam case i) 
Graphs in Figure 8 show the fitness for the case ii). Mean fitness values for frequencies of 3,5 , and $7 \mathrm{~Hz}$ are $2.56,2.73$, and 1.97 , respectively. In this case, the frequency of $5 \mathrm{~Hz}$ is a good choice for the tuning of the Contouring impedance parameter since it gives the best response in most cases.
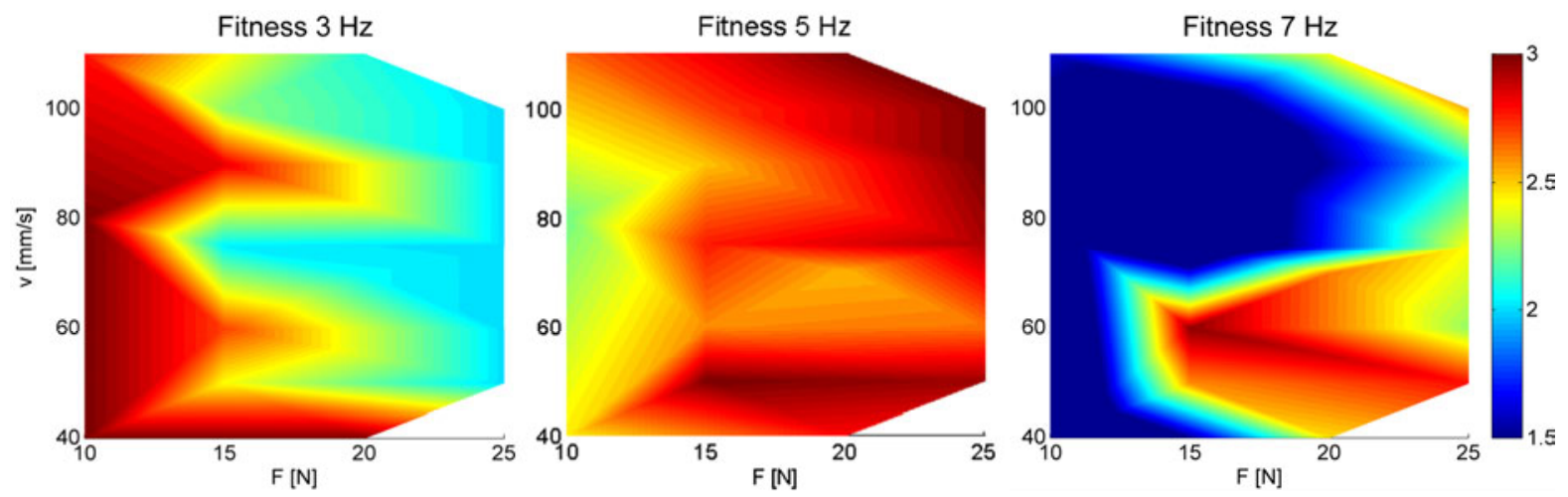

Fig. 8 Fitness of frequencies for the rigid flat beam case ii)

When these results are compared to the case i), one can conclude that a more rigid structure demands a lower frequency to reduce the vibrations of the whole system. A decrease in frequency causes larger force deviations when the tool is far from the rigid support, which is shown by the standard deviation calculated for the cases using frequencies of 5 and $7 \mathrm{~Hz}$ (see Figure 9).

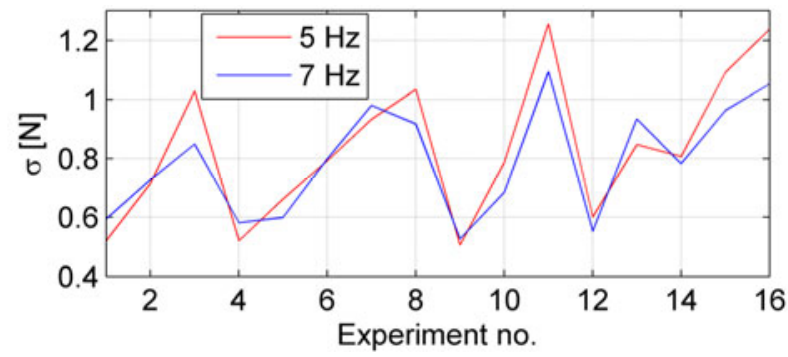

Fig. 9 Standard deviation for the case ii) and the tool far away from the rigid support

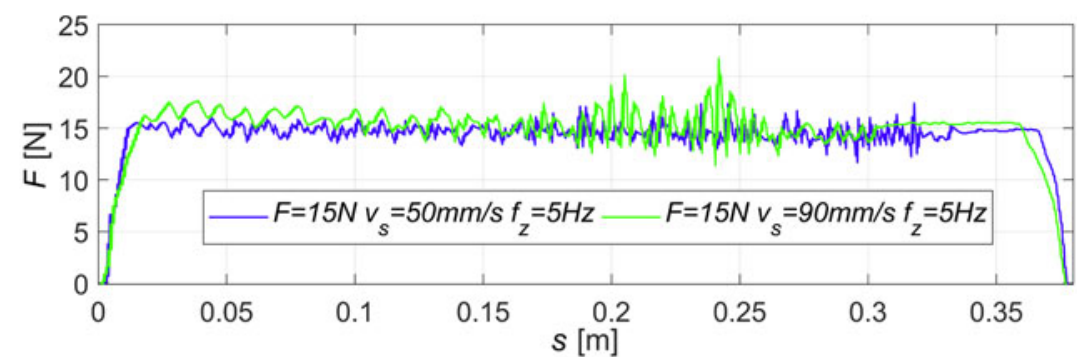

a)

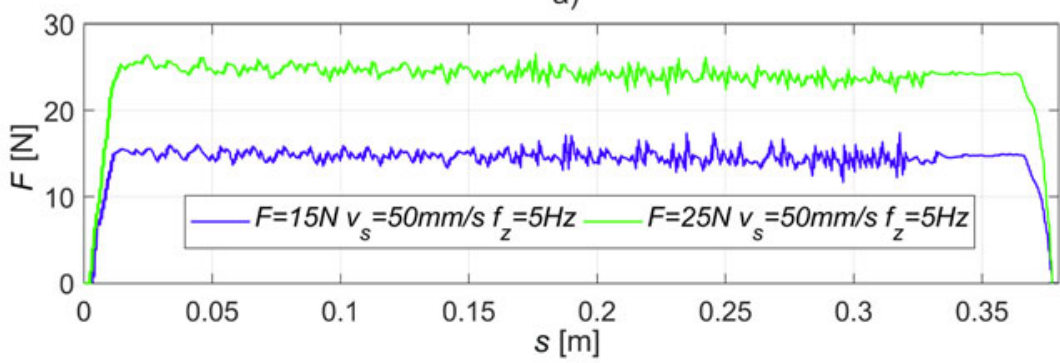

b)

Fig. 10 Experimental results for the flat beam case ii) 
Graphs in Figure 10a) show the impact of the feed rate on the force response. Responses with different feed rates for equal target forces $(F=25 \mathrm{~N})$ and impedance frequencies $\left(f_{\mathrm{z}}=5 \mathrm{~Hz}\right)$ can be noted. It can be seen that with an increase in the feed rate value, the oscillations in the values of the exerted force also increase. The impact of the target force on the response is shown by the graph in Figure $10 \mathrm{~b}$ ). Responses with different target forces for equal feed rates $\left(v_{\mathrm{s}}=50 \mathrm{~mm} / \mathrm{s}\right)$ and impedance frequencies $\left(f_{\mathrm{z}}=5 \mathrm{~Hz}\right)$ can be noted. The responses show almost equal absolute force errors (equation 1) for both target forces, which means bigger relative force errors for lower values of target forces. Better responses can be observed at higher values of target forces.

Workpieces which are more compliant provide better damping of vibrations caused by the rise in the contouring frequency. Since a lower value of contouring frequency yields sluggish responses, it is important to determine the frequency which fits the compliance of the workpiece and the tool and gives satisfactory results. Moreover, a compliant tool would give lower force oscillations during robot contouring.

\subsection{Wave shaped compliant plate test, case iii)}

Experimental tests for the wave-shaped compliant plate were carried out for force reference values of $10,15,20$, and $25 \mathrm{~N}$, while the deviation of the force was observed for the feed rates of $5,10,20,25,30,35,40,50$, and $60 \mathrm{~mm} / \mathrm{s}$. Since the robot tool and the steel waved shaped plate are both rigid, it was not possible to conduct tests with the steel plate mounted directly onto the aluminum work table as this caused very powerful vibrations. To reduce the vibrations, soft inserts with a thickness of $10 \mathrm{~mm}$ were empirically chosen and assembled, as previously mentioned in section 2.1 . The tests were conducted with frequency changes along the Z-axis of the tool coordinate system for each feed rate and force. Graphs in Figure 11 show the fitness for frequencies of 10,15 , and $20 \mathrm{~Hz}$ for all the feed rates and forces applied. The mean fitness values for frequencies of 10,15 , and $20 \mathrm{~Hz}$ are 2.08, 2.67, and 2.34, respectively. It has been shown that the frequency of $15 \mathrm{~Hz}$ in the $\mathrm{Z}$-axis direction is a good choice for the tuning of the Contouring impedance parameter. This frequency gives the best response with minimum vibrations of the robotic system in most cases.
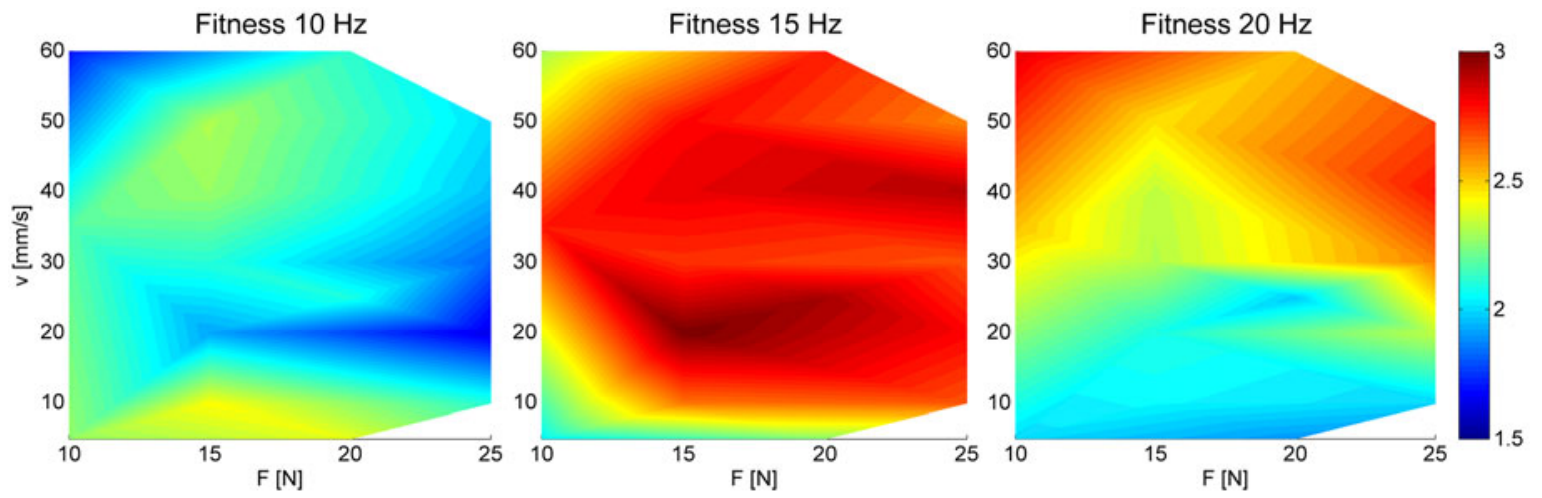

Fig. 11 Fitness of frequencies for the wave shaped plate

Experimental tests gave similar results to those of the flat beam tests considering the impact of the target forces and feed rates on the force response with an exception of very low feed rates. This exception may be a result of the noise of the measured signal since the tool moves very slowly along the predefined path. The force exerted between the tool and the workpiece does not change significantly over a short period of time because of the workpiece curvature. The signal noise gives false changes in the force, which makes the robot react when it is not needed and causes vibrations of the whole system. This problem was beyond the scope of this research but it should be further examined. 


\subsection{Compliant flat plate test, case iv)}

The appearance of vibrations was noted during experiments when the robot tool tip was further away from the robot base. Considering this observation, a simple compliant flat plate was used to examine the impact of workpiece position in the robot workspace on the contouring performance. Although, it would be interesting to compare the compliant flat plate case and the cases with the flat beam, it would require additional measurements which were left out of the scope of this research. The impact of the workpiece position was tested by placing a compliant flat plate very close to the robot base (Figure 4 left, starting point $490 \mathrm{~mm}$ away from the origin of the robot base coordinate system) and on the edge of the robot workspace (Figure 4 right, starting point $900 \mathrm{~mm}$ away from the origin of the robot base coordinate system). Tests were performed with an experimental frequency of $5 \mathrm{~Hz}$, which gave good results for the case ii). Figure 12 shows results for different positions of the workpiece. The force response is better when the plate is near the robot base (blue line) than in the case when the plate is far away from the robot base (green line).

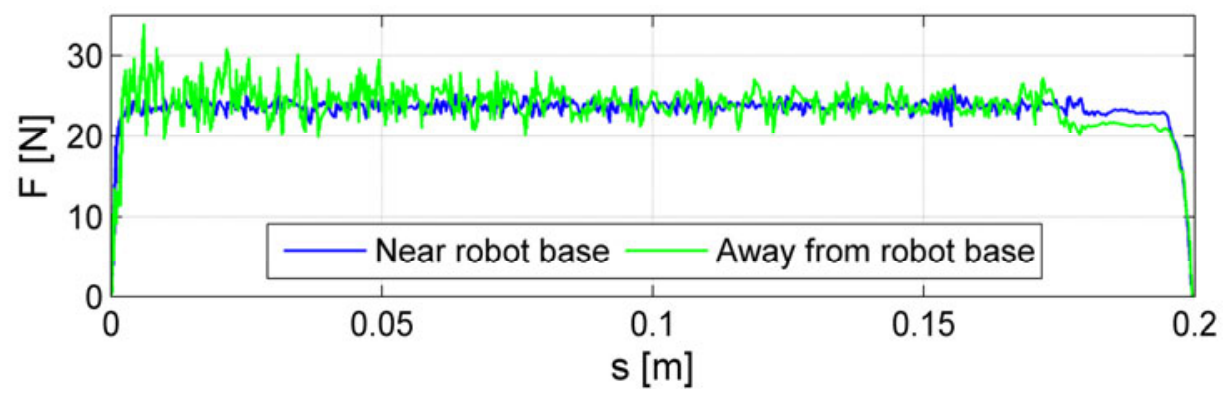

Fig. 12 Results for the compliant flat plate

Lower oscillations were obtained for the case when the plate was near the robot base. For $F_{0}=25 \mathrm{~N}$, the mean force value and the standard deviation was $\bar{F}=23.73 \pm 0.63 \mathrm{~N}$, and $\min / \max$ errors were $-3.78 \mathrm{~N}$ and $+1.45 \mathrm{~N}$, respectively. The positioning of the workpiece has a big impact on contouring performance and should be further examined for every specific case. We can conclude that the workpiece should be as close as possible to the robot base because of higher rigidity of the robot arm.

\section{CONCLUSION AND FUTURE WORK}

The integration of advanced sensor systems into a robotic system enables various tasks to be performed in industrial production and increases the robot flexibility and efficiency. An additional very important aspect is an increase in the quality of the task performance. In this paper, we have tested the application of robot force contouring in cases when a constant normal force is applied to the surface along a predefined path, which is usually the case in robotic grinding or polishing. A fitness function for evaluating and validating the Contouring impedance parameter based on statistical values of force deviation is proposed and used for tuning the Contouring impedance parameter. Although our measurements showed that there is no single solution for all applications, they pointed to useful conclusions. We have concluded that in the case of flat surfaces, the best initial setting of the Contouring impedance parameter in the case with a rigid support is a lower frequency $(5 \mathrm{~Hz})$ and in the case of a wave-shaped compliant plate without a support, higher frequencies $(7 \mathrm{~Hz})$. This is in a direct relation to stiff surfaces which amplify vibrations and a "softer" surface which provides the damping effect. For the curved surfaces, the best initial setting of the Contouring impedance parameter was a frequency of $15 \mathrm{~Hz}$. Depending on the applied forces, feed rates, and workpiece stiffness, it is necessary to further fine-tune the Contouring function control parameters, 
especially the Contouring impedance parameter. The response error significantly decreases when the workpiece is close to the robot base and increases when the workpiece is on the edge of the robot workspace.

The introduction of a compliant tool $[8,21,22,23,24]$, which was not considered in our paper, could provide us with new insights to be used in our research. In future work, we also plan to conduct a series of measurements by developing and testing a compliant robot tool for exerting a constant normal force. Furthermore, we plan to use the design of experiments for the assessment of the relationship between the environmental (damping, stiffness), the controller (impedance parameters), and the response (force deviation) parameters in order to optimize the controller parameters and reduce vibrations and force deviation. Another possible approach which will be taken into consideration is the development of our own controller for contouring applications whose structure and parameters would be known in detail since many studies have shown improved performance by using advanced control algorithms $[7,10,11,13,16,17,21]$. We also intend to use the results from this research in the development of robotic applications for sensitive bone drilling [27].

\section{Acknowledgements}

The authors would like to acknowledge the support of the Croatian Scientific Foundation through the research project ACRON - A new concept of Applied Cognitive RObotics in clinical Neuroscience.

\section{REFERENCES}

[1] E. Guizzo and E. Ackerman, "The rise of the robot worker," IEEE Spectrum, vol. 49, no. 10, 2012. https://doi.org/10.1109/MSPEC.2012.6309254

[2] F. Šuligoj, B. Šekoranja, M. Švaco, and B. Jerbić, “Object Tracking with a Multiagent Robot System and a Stereo Vision Camera,” Procedia Engineering, vol. 69, pp. 968-973, 2014. https://doi.org/10.1016/j.proeng.2014.03.077

[3] I. Lopez-Juarez and M. Howarth, "Learning manipulative skills with art," in Intelligent Robots and Systems, 2000.(IROS 2000). Proceedings. 2000 IEEE/RSJ International Conference on, 2000, vol. 1, pp. 578-583.

[4] M. Švaco, D. Bašić, B. Šekoranja, and B. Jerbić, "A capacitive sensor fo human-robot interaction," Annals of DAAAM for 2012. \& Proceedings of the 23rd International Symposium, vol. 23, pp. 819-822, 2012.

[5] M. Mronek, J. Barta, "Inaccuracies of industrial robot positioning and methods of their correction," Tehnicki vjesnik - Technical Gazette, vol. 22, no. 5, Oct. 2015.

[6] M. Švaco, B. Šekoranja, F. Šuligoj, and B. Jerbić, "Calibration of an Industrial Robot Using a Stereo Vision System,” Procedia Engineering, vol. 69, pp. 459-463, 2014.

https://doi.org/10.1016/j.proeng.2014.03.012

[7] C. Y. Lai, "Improving the transient performance in robotics force control using nonlinear damping," in Advanced Intelligent Mechatronics (AIM), 2014 IEEE/ASME International Conference on, 2014, pp. 892-897.

[8] C. Li, Z. Wang, C. Fan, G. Chen, and T. Huang, "Research on Grinding and Polishing Force Control of Compliant Flange," MATEC Web of Conferences, vol. 22, p. 03012, 2015.

[9] Y. TIAN et al., "Research on layout and operational pose optimization of robot grinding system based on optimal stiffness performance," Journal of Advanced Mechanical Design, Systems, and Manufacturing, vol. 11, no. 2, pp. JAMDSM0022-JAMDSM0022, 2017.

[10] I. Bonilla, E. Gonzalez-Galvan, M. Mendoza, C. Chavez-Olivares, A. Loredo-Flores, and F. Reyes, "Force-Contouring Process Using Uncalibrated Vision and Impedance Control," IFAC Proceedings Volume, vol. 43, no. 4, pp. 296-301, 2010.

[11] S.-J. Huang, C.-K. Yu, and J.-Y. Lin, "Intelligent robotic impedance control using embedded system structure," Transactions of the Institute of Measurement and Control, vol. 35, no. 5, pp. 561-573, Jul. 2013. https://doi.org/10.1177/0142331212460718 
[12] K.-H. Low, Ed., Industrial robotics: programming, simulation and applications. Mammendorf: plV, proLiteratur-Verl, 2007.

[13] A. Winkler and J. Suchy, "Force controlled contour following on unknown objects with an industrial robot," in Robotic and Sensors Environments (ROSE), 2013 IEEE International Symposium on, 2013, pp. 208-213.

[14] A. M. Tahvilian, B. Hazel, F. Rafieian, Z. Liu, and H. Champliaud, "Force model for impact cutting grinding with a flexible robotic tool holder," The International Journal of Advanced Manufacturing Technology, vol. 85, no. 1-4, pp. 133-147, Jul. 2016. https://doi.org/10.1007/s00170-015-7919-z

[15] Y. Sun, D. J. Giblin, and K. Kazerounian, “Accurate robotic belt grinding of workpieces with complex geometries using relative calibration techniques," Robotics and Computer-Integrated Manufacturing, vol. 25, no. 1, pp. 204-210, Feb. 2009. https://doi.org/10.1016/j.rcim.2007.11.005

[16] X. Xie and L. Sun, "Force control based robotic grinding system and application," Intelligent Control and Automation (WCICA), 2016 12th World Congress on, pp. 2552-2555, 2016.

[17] J. Zhang, G. Liu, X. Zang, and L. Li, "A hybrid passive/active force control scheme for robotic belt grinding system," in Mechatronics and Automation (ICMA), 2016 IEEE International Conference on, 2016, pp. 737-742.

[18] W. Chen, J. M. Zhan, and M. Q. Zhang, "Study on Polishing Tool Contact Deformation for Large Robotic Aspheric Surface Compliant Polishing," Applied Mechanics and Materials, vol. 433-435, pp. 2058-2063, Oct. 2013. https://doi.org/10.4028/www.scientific.net/AMM.433-435.2058

[19] R. Zamri, N. Ismail, B. T. H. T. Baharudin, M. A. Azmah Hanim, and A. S. Prabuwono, "Analysis of Flat and Dry Polishing Parameter Influence on Surface Roughness for Robotic Polishing Cell," Applied Mechanics and Materials, vol. 564, pp. 543-548, Jun. 2014. https://doi.org/10.4028/www.scientific.net/AMM.564.543

[20] A. E. Khalick Mohammad, J. Hong, and D. Wang, "Polishing of uneven surfaces using industrial robots based on neural network and genetic algorithm," The International Journal of Advanced Manufacturing Technology, vol. 93, no. 1-4, pp. 1463-1471, Oct. 2017. https://doi.org/10.1007/s00170-017-0524-6

[21] H. Du, Y. Sun, D. Feng, and J. Xu, “Automatic robotic polishing on titanium alloy parts with compliant force/position control," Proceedings of the Institution of Mechanical Engineers, Part B: Journal of Engineering Manufacture, vol. 229, no. 7, pp. 1180-1192, Jul. 2015. https://doi.org/10.1177/0954405414567518

[22] Amtru Business AG Wiesendangen, "Sonderwerkzeug und entgraten mit Roboter - Amtru Business AG." [Online]. Available: http://www.amtru.com/robotapplications-tools. [Accessed: 07-Nov-2017].

[23] “ACF," Ferro Local, 14-Apr-2017. [Online]. Available: http://www.ferrobotics.com/en/technologieprodukte/produkte/acf/. [Accessed: 07-Nov-2017].

[24] A. E. K. Mohammad and D. Wang, "A novel mechatronics design of an electrochemical mechanical endeffector for robotic-based surface polishing," in System Integration (SII), 2015 IEEE/SICE International Symposium on, 2015, pp. 127-133.

[25] S. Huang, N. Bergstrom, Y. Yamakawa, T. Senoo, and M. Ishikawa, "Robotic Contour Tracing with High-Speed Vision and Force-Torque Sensing based on Dynamic Compensation Scheme," 2017.

[26] M. Švaco, N. Vitez, B. Jerbić, F. Šuligoj, B. Šekoranja, and J. Vidaković, "Experimental Evaluation of Parameters for Robotic Contouring Force Feedback Applications," presented at the The International Conference Management of Technology - Step to Sustainable Production (MOTSP 2017), Dubrovnik, Croatia, 2017.

[27] M. Švaco, B. Šekoranja, F. Šuligoj, J. Vidaković, B. Jerbić, and D. Chudy, “A Novel Robotic Neuronavigation System: RONNA G3," Strojniški vestnik - Journal of Mechanical Engineering, Nov. 2017. https://doi.org/10.5545/sv-jme.2017.4649

Submitted: $\quad 30.01 .2018$

Accepted: $\quad 08.5 .2018$
Marko Švaco

Nikola Vitez

Bojan Šekoranja*

Filip Šuligoj

University of Zagreb, Faculty of

Mechanical Engineering and Naval

Architecture,

Ivana Lučića 5, Zagreb, Croatia

bojan.sekoranja@fsb.hr 